\title{
The effect of the number of stool samples on the observed prevalence and the infection intensity with Schistosoma mansoni among a population in an area of low transmission
}

\author{
Martin Johannes Enk ${ }^{\mathrm{a}, *}$, Anna Carolina Lustosa Lima ${ }^{\mathrm{b}}$, Sandra Costa Drummond ${ }^{\mathrm{c}}$, \\ Virginia Torres Schall ${ }^{\mathrm{d}}$, Paulo Marcos Zech Coelho ${ }^{\mathrm{a}}$ \\ a Laboratory of Schistosomiasis, René Rachou Research Center/Oswaldo Cruz Foundation, Av. Augusto de Lima 1715, Belo Horizonte, Minas Gerais, Brazil \\ ${ }^{\mathrm{b}}$ Department of Scientific Support, Unit for Biostatistics, René Rachou Research Center/Oswaldo Cruz Foundation, Av. Augusto de Lima 1715, Belo Horizonte, Minas Gerais, Brazil \\ ' Secretary of Health in the State of Minas Gerais, Rua Rio Grande do Norte 613, Belo Horizonte, Minas Gerais, Brazil \\ ${ }^{\mathrm{d}}$ Laboratory of Health Education, René Rachou Research Center/Oswaldo Cruz Foundation, Av. Augusto de Lima 1715, Belo Horizonte, Minas Gerais, Brazil
}

\section{A R T I C L E I N F O}

\section{Article history:}

Available online 5 October 2008

\section{Keywords:}

Schistosomiasis

Diagnosis

Kato Katz technique

Prevalence

Infection intensity

Control

Low endemic areas

Brazil

\begin{abstract}
A B S T R A C T
Examination of faecal material by Kato Katz (KK) technique is a widely used approach for the diagnosis of intestinal schistosomiasis, particularly in epidemiological surveys. However, this technique lacks diagnostic sensitivity in individuals with low infection intensity or in low endemic areas. In the total population (TP) of 1265 individuals prevalence and infection intensity were established by examining two KK slides. A representative subset of 305 individuals, denominated experimental group (EG), was defined to assess the comparative advantage of an increased sampling effort using the KK technique. In addition stools of the participants of the EG were examined by the formol ether centrifugation technique. The proportion of all positive stool examinations detected by both methods among the experimental group served as reference value for prevalence (diagnostic 'gold' standard). Prevalence of schistosomiasis among TP based on two KK slides was $12.5 \%$. Prevalence among the EG varied from 13.8\%, based on one KK slide, over 27.2 based on $10 \mathrm{KK}$ slides of three stool samples to $35.4 \%$ as value for the diagnostic 'gold' standard. The comparison of values for prevalence, stratified by age, revealed significant elevated numbers for all age groups, and interestingly, an extension of the highest prevalence levels until an age of 50 years. The overall infection intensity in eggs per gram (epg), calculated as geometric mean, was 83 epg for the TP based on one KK slide, 78 epg for the GE based on one KK slide and 28 epg based on $10 \mathrm{KK}$ slides of three stool samples. In summary our data demonstrate that control programmes based on the examination of a single KK slide, as it is the case in Brazil, tend to underestimate significantly the prevalence and overestimate infection intensity. This applies especially for low endemic areas, where the efficacy and cost-effectiveness of such programmes become questionable. Our data also indicate that the possible solution of this problem lies in targeted mass treatment including age groups with the highest proportions of those infected. This will give high sensitivity together with sustainability and suitability under field conditions.
\end{abstract}

(c) 2008 Elsevier B.V. All rights reserved.

\section{Introduction}

Infection with Schistosoma mansoni is one of the major parasitic diseases in the tropics and subtropics. Its socio-economic and public health importance based on symptoms and disability linked morbidities such as anaemia, chronic pain, diarrhoea, exercise intolerance and undernutrition is evident (King et al., 2005). Recent estimates of 779 million people at risk and 207 million infected for all schistosomiasis species, indicates an increase of $12.6 \%$ and $7.2 \%$,

\footnotetext{
* Corresponding author. Tel.: +55 31 33497700; fax: +55 3132953115 .

E-mail address: marenk@cpqrr.fiocruz.br (M.J. Enk).
}

respectively in comparison to figures of the last decade (Chitsulo et al., 2000; Steinmann et al., 2006,). In Brazil the number of infected is estimated between 2.5 and 6 million for $S$. mansoni, which is the only species transmitted in this country (Passos and Amaral, 1998; Katz and Peixoto, 2000). The diagnosis of the disease is usually carried out by parasitological methods. The technique of Kato (Kato and Miura, 1954), modified by Katz et al. (1972), is the procedure of choice recommended by the World Health Organization (WHO) for the detection of eggs in faeces (WHO, 1994). It is used on a large scale for identification and quantification of the disease on individuals and at population level because of its practical advantages and good performance in diagnosing moderate and heavy infections. Nevertheless, it is a well-known fact and also scientifically 
well documented that this method is limited in the detection of positive individuals, when intensity of infection is low and it is less reliable in determining the prevalence in low endemic areas (Feldmeier and Poggensee, 1993; Kongs et al., 2001). In order to overcome these shortcomings, an increase in number of stool samples as well as in the number of slides per specimen is suggested. There exists general consensus that two KK slides for each of three stool samples improve enough sensitivity to obtain reliable results (Barreto et al., 1990; Rabelo et al., 1992; Engels et al., 1996, 1997; Utzinger et al., 2001).

In the present study we assessed prevalence and infection intensity among a population in a low-endemic area with two KK slides of a single stool sample. In a representative subset of this population we evaluated the diagnostic performance, examining increasing numbers of stool samples and slides, starting with $1 \mathrm{KK}$ smear up to 10 smears of three stool samples. Additionally, stool samples were examined by the formol ether centrifugation technique described by Blagg et al. (1955) in order to establish a solid reference value for prevalence, called diagnostic 'gold' standard. The variation of prevalence and infection intensity is described, and the implication on the decision-making process for individual treatment and the impact on control programmes are discussed based on these data.

\section{Materials and methods}

\subsection{Study area and population}

The present work is a cross-sectional study of a population and a representative subset for a semi-urban community in the state of Minas Gerais, Brazil. The village, Chonim de Cima, is located in the county of Governador Valadares, $30 \mathrm{~km}$ distant from the county's main city. Out of the 1448 inhabitants of the village, 1265 participated in the study (183 were excluded because they were not permanent residents of the village). A systematic random sample of 305 individuals was included in the investigation in order to reduce the huge number of required stool samples for the in-depth analysis of the objectives. The sample size was calculated based on a test on comparison of proportions with a level of confidence of $95 \%$ and a power of the test of $90 \%$. The detected prevalence of the population (13\%), the expected prevalence of the population (22\%) and the size of population (1265) was used to calculate the minimum sample size of 289 individuals. This experimental group (EG) consisting of 305 individuals was representative of the total population in relation to age (chi-square test: $p=0.147$ ) and sex (chi-square test: $p=0.137$ ). Furthermore, no statistically significant difference was found between the total population (TP) and the experimental group for the distribution of $S$. mansoni positive and negative individuals, obtained through the examination of two slides of one stool sample according to the KK technique (chi-square test: $p=0.174$ )

\subsection{Field and laboratory procedures}

All 1265 participants were asked to provide one stool sample. Two KK smears were prepared from this single stool sample. The 305 participants of the experimental group were asked to provide a total number of four stool samples during a period of 2 weeks. Six KK smears were prepared from the first stool sample and two smears from the second and the third sample. Only 264 participants returned the fourth stool sample of which 66 samples provided enough material for six smears, 1 for five slides, 196 for two slides and 1 for one slide. Additionally $0.5 \mathrm{~g}$ of 452 stool samples of the experimental group was processed by the formol ether centrifugation technique and the total sediment was examined. A number of 218 samples of the first stool specimen were examined according to
Table 1a

Summary of all Kato Katz smears of all stool samples examined and considered for the value of the diagnostic 'gold' standard.

\begin{tabular}{lr}
\hline Number of smears (Kato Katz) & Number of participants \\
\hline 10 smears of three samples (10 sts) & 41 \\
10 sts +1 slide of the fourth sample & 1 \\
10 sts +2 slides of the fourth sample & 196 \\
10 sts +5 slides of the fourth sample & 1 \\
10 sts +6 slides of the fourth sample & 66 \\
Total & 305 \\
Total number of smears & 3844 \\
\hline
\end{tabular}

the technique and used for direct comparison with six KK smears of the same specimen. A combination of all results of both techniques served as diagnostic 'gold' standard for the experimental group (Tables $1 \mathrm{a}$ and $1 \mathrm{~b}$ ).

Approximately $10 \%$ of all slides were subjected to a control system for diagnostic efficacy.

For each individual the arithmetic mean egg count per gram based on the KK samples was calculated. For analysis of groups the intensity of infection was calculated as the geometric mean of the individual egg counts.

\subsection{Ethical considerations and treatment}

The study objectives were explained to the participants and informed written consent was obtained from the study population. In case of children below the age of 16 years the consent form was signed by the legal representative.

The study protocol was received and approved by the Ethical Review Board of the René Rachou Institute/Fiocruz (No. 04/2005)

All participants identified as positive for schistosomiasis were treated with praziquantel (adults, $50 \mathrm{mg} / \mathrm{kg}$ body weight in a single dose; children $\leq 15$ years of age $60 \mathrm{mg} / \mathrm{kg}$ body weight in a single dose).

\subsection{Statistical analysis}

The chi-square test was used to compare proportions between groups and the method of Bonferroni in case of multiple comparisons. For comparison of infection intensity of positive individuals according to number of slides and stool samples the paired test of Wilcoxon was applied and the Mann-Whitney test for the comparison of the overall infection intensity between the total population and the experimental group. The level of concordance of results obtained by the KK technique and the formol ether centrifugation technique was evaluated by Kappa statistics. The level of concordance is categorized as bad $(<0.20)$, weak $(0.21-0.40)$, moderate (0.41-0.60), good (0.61-0.80) and excellent (0.81-1) (Landis and Koch, 1977). Test results are reported together with $95 \%$ confidence intervals. The software package MINITAB 14.0 was used to carry out these calculations.

\section{Table 1b}

Summary of all stool samples examined by the formol ether centrifugation technique described by Blagg et al. and considered for the value of the diagnostic 'gold' standard.

\begin{tabular}{lc}
\hline Number of samples (Blagg et al.) & Number of participants \\
\hline 0 (no stool sample returned) & 18 \\
1 & 137 \\
2 & 135 \\
& 15 \\
Total & 305 \\
Total number of samples & 452 \\
\hline
\end{tabular}




\section{Results}

A total of 1265 individuals (693 females and 572 males) participated in the survey to establish the prevalence of schistosomiasis within the community. There were 142 positives ( 40 females and 102 males) with one KK smear and 158 positives (47 females and 111 males) with two KK smears of the same stool sample resulting in prevalence estimates of $11.2 \%$ and $12.5 \%$, respectively. Among the 305 participants ( 181 females and 124 males) of the experimental group a number of 42 ( 14 females and 28 males) individuals, corresponding a prevalence of $13.7 \%$, proofed positive for schistosomiasis and 47 ( 15 females and 32 males), resulting in a prevalence of $15.4 \%$, by applying the same methodology as for the entire population. The comparison of the proportions of positives, as well as their sex related proportions between the experimental group and population showed no statistical significant difference (total proportions of all positives: chi-square test for one KK smear: $p=0.215$ and for two KK smears: $p=0.174$ : sex related proportions of all positives: chi-square test for one KK smear: $p=0.518$ and for two KK smears: $p=0.776$ ), which indicates that data obtained from the experimental group are representative for the total population.

\subsection{Effect of sampling effort on infection prevalence}

The increase of one KK smear to six KK smears of a single stool sample among the experimental population shows an increase of prevalence estimates from $13.7 \%$ to $19 \%$. The increase of number of stool samples resulted - as expected - in a further increase of prevalence estimates from $20.7 \%$ to $27.2 \%$. A proportion of $35.4 \%$ of positive individuals was found considering all stool examinations carried out among the participants of the EG. This proportion reflects the closest value to the real prevalence and was used as diagnostic 'gold' standard for comparisons. Table 2 gives more details about the figures related to the increase of KK smears and stool samples, as well as their comparison to the "diagnostic 'gold' standard".

Fig. 1 illustrates the prevalence resulting from examination of one single KK smear and the values obtained for the diagnostic 'gold' standard, stratified by seven age groups. The findings indicate, as expected, the highest prevalence among children and young adults (range of age from 10 to 30 years), followed by a continuous decrease of the proportion of infected with increasing age, in case of the use of a single KK smear. In case of the values for the diagnostic 'gold' standard, besides the general increase of prevalence for each single age group, the highest levels of prevalence are maintained from 10 to 50 years of age.

The comparison of the overall infection intensity between the total population and the experimental group showed values of 83 and 78 epg using one KK slide of the first sample. No significant

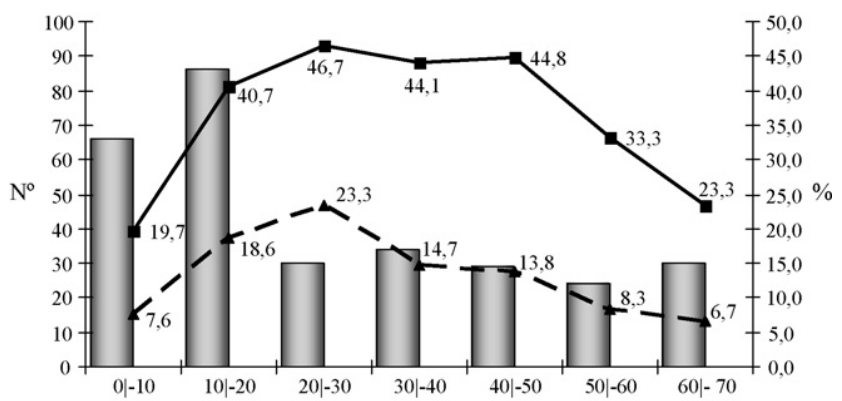

Number of individuals $\longrightarrow \longleftarrow 1$ slide $\longrightarrow-$ diagnostic 'gold' standard

Fig. 1. Variability of the proportion of schistosomiasis positive individuals identified with one Kato Katz smear of a single sample or by the diagnostic 'gold' standard in relation to different age groups.

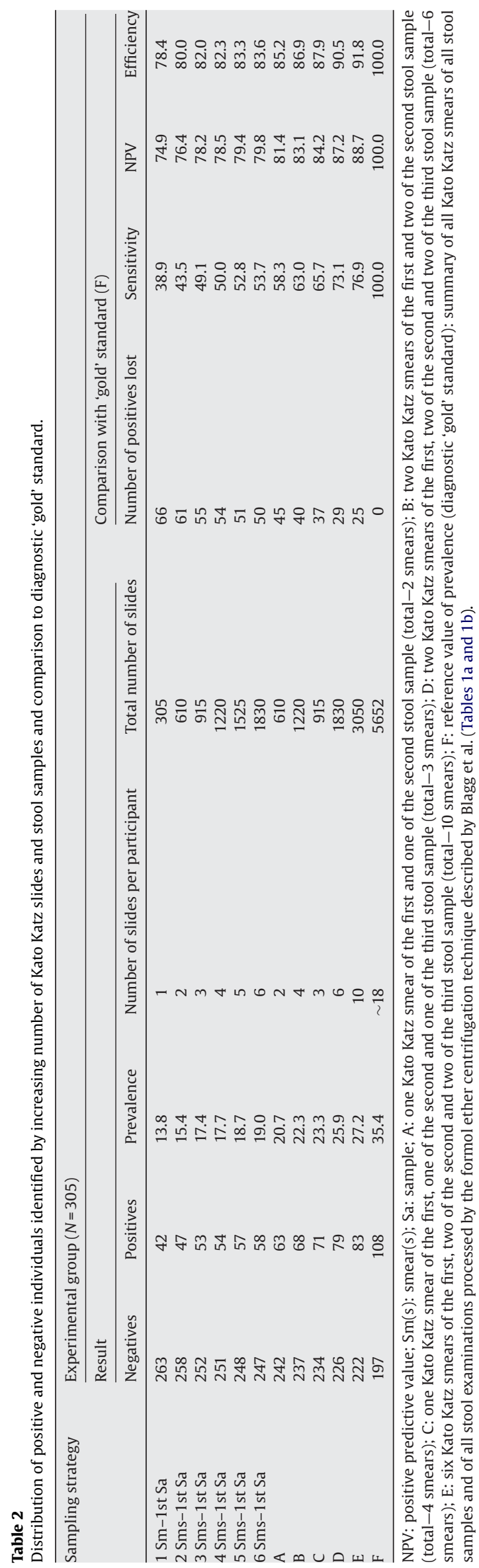


Table 3

Number of positive individuals identified with one Kato Katz slide or ten slides of three samples categorized by infection intensity.

\begin{tabular}{|c|c|c|c|c|}
\hline \multirow[t]{2}{*}{ Infection intensity } & \multicolumn{4}{|c|}{ Ten slides } \\
\hline & Low & Medium & High & Total \\
\hline \multicolumn{5}{|l|}{ One slide } \\
\hline Low & 23 & 5 & 0 & 28 \\
\hline Medium & 0 & 7 & 1 & 8 \\
\hline High & 0 & 2 & 4 & 6 \\
\hline Total & 23 & 14 & 5 & 42 \\
\hline
\end{tabular}

difference between these values was found according to the Mann-Whitney test $(p=0.555)$.

\subsection{Effect of sampling effort on infection intensity}

The overall infection intensity of the 42 positives detected with one KK slide among the participants of the experimental group revealed no significant change in geometric mean egg count per gram, when examined with one single slide or with 10 slides of three samples (78 epg, 1 slide; $81 \mathrm{epg}, 10$ slides three samples; Wilcoxon's test: $p=0.339$ ). At a closer look, the stratification of these 42 positives into three categories of infection intensity (low $=1-100 \mathrm{epg}$, medium $=101-400$ epg and high $\geq 401 \mathrm{epg}$ ) according to the results of one KK slide and 10 slides of tree samples shows that a total number of eight positives migrated between the categories, five from category low to medium, two from high to medium and one from medium to high (Table 3 ).

In contrary to the above-mentioned findings, the overall infection intensity, revealed declining figures when the geometric mean egg count of all positives detected with one or $10 \mathrm{KK}$ slides are compared. The geometric mean egg count obtained among the 42 positives with one slide was $78 \mathrm{epg}$ and a value of $28 \mathrm{epg}$ was registered for the 83 positives with 10 slides of three samples. It should be noted that the calculation of the geometric mean egg count in this case is based on all positives detected with one or $10 \mathrm{KK}$ slides, which resulted in an inclusion of additional 42 positives.

As shown in Table 4, the results for infection intensity are influenced by sample size and number of slides examined. With regard to infection intensity categories a sharp increase in the number of infected individuals, especially a in the group of low egg counts was registered. A total number of 28 positives were detected with one single slide, and 61 individuals were found positive, using six slides of the first and two slides of the second and third stool specimen. This means an increase of 33 positives or $117.9 \%$ in the category of low infection intensity. In the category of medium infection intensity (101-400 epg) a significant increase from 8 to 17 positive individuals was found. The reduction of one positive in category of high infection intensity was caused by migration to next lower level as described above. In summary the differences between the com-
Table 5

Comparison of the total number of participants negative and positive for schistosomiasis identified by the formol ether centrifugation method and by three smears of the same stool sample according to the technique of Kato Katz.

\begin{tabular}{llcc}
\hline Blagg & \multicolumn{4}{l}{ Kato Katz (three smears) } & Total \\
\cline { 2 - 4 } & Positive & Negative & 67 \\
\hline Positive & 49 & 18 & 151 \\
Negative & 18 & 133 & 218 \\
Total & 67 & 151 &
\end{tabular}

parisons of both examination procedures in relation to infection intensity categories consist in total increase of 41 positive individuals and in the migration of 8 individuals.

Comparing infection intensity related to age groups, the highest was found among children below 10 years of age for 1 slide as well as for 10 slides, but again the geometric mean egg count decreases when evaluated with 1 or 10 slides. The findings of this age group have to be observed with caution because of the small sample size. In the same age group of the total population the evaluation with one KK slide the overall infection intensity also showed the highest value (mean: $154 \mathrm{epg}$, locating four individuals in the infection intensity category low, four in category medium and two in category high), which makes the results of the experimental group up to a certain extent more reliable. In the other age groups of the experimental group a reduction of geometric mean egg count was also observed according to the increase of slides and stool samples.

With regard to the direct comparison between the qualitative analysis of stool samples according to the KK technique and the formol ether centrifugation method among 218 participants, the prevalence of infection in this group was $30.7 \%$ which equals exactly the results of three KK smears of a single stool sample. Both techniques identified 67 individuals as positive for schistosomiasis (Table 5). A more profound analysis of the results reveals that both methods jointly identify a total number of 85 (100\%) positive individuals. Both methods detect 67 (78.8\%) of infected, but only 49 (57.6\%) of them are identified by each method. Of the remaining 36 (42.4\%) each method detects 18 (21.2\%) separately. The coefficient of concordance, Kappa, reveals a value of 0.612 , which indicates moderate to good strength of concordance. A combination of both methods reveals an increase in prevalence of $21.2 \%$ in this setting of 218 participants.

\section{Discussion}

Our data confirm and strongly support that prevalence of S. mansoni in areas of low transmission using only a single KK slide is considerable underestimated (de Vlaas and Gryseels, 1992). Extrapolating our results of the experimental group, which establishes a prevalence of $35.4 \%$ as reference value, to the total population of

Table 4

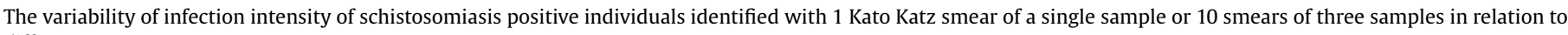
different age groups.

\begin{tabular}{|c|c|c|c|c|c|c|c|c|c|c|c|c|}
\hline \multirow[t]{3}{*}{ Age group } & \multicolumn{6}{|c|}{ One Kato Katz slide of one sample } & \multicolumn{6}{|c|}{ Ten Kato Katz slides of three samples ${ }^{a}$} \\
\hline & \multicolumn{2}{|l|}{ Result } & \multicolumn{3}{|c|}{ Infection intensity ${ }^{\mathrm{b}}$} & \multirow[t]{2}{*}{ Geometric mean } & \multicolumn{2}{|l|}{ Result } & \multicolumn{3}{|c|}{ Infection intensity ${ }^{\mathrm{b}}$} & \multirow{2}{*}{$\begin{array}{l}\text { Geometric } \\
\text { mean }\end{array}$} \\
\hline & Negative & Positive & Low & Medium & High & & Negative & Positive & Low & Medium & High & \\
\hline $0-10$ & 61 & 5 & 1 & 2 & 2 & 241 & 57 & 9 & 4 & 3 & 2 & 58 \\
\hline $10-30(10-50)$ & 93 (147) & $23(32)$ & $17(23)$ & $3(5)$ & $3(4)$ & $67(68)$ & 78 (119) & $38(60)$ & $28(45)$ & $8(12)$ & $2(3)$ & $37(31)$ \\
\hline$\geq 30(\geq 50)$ & $109(55)$ & $14(5)$ & $10(4)$ & $3(1)$ & $1(0)$ & $67(59)$ & $87(46)$ & $36(14)$ & $29(12)$ & $6(2)$ & $1(0)$ & $18(11)$ \\
\hline Total & 263 & 42 & 28 & 8 & 6 & 78 & 222 & 83 & 61 & 17 & 5 & 28 \\
\hline
\end{tabular}

a Six Kato Katz slides of the first, two of the second and two of the third stool sample (total-10 slides).

b Low $=1-100$ eggs per gram, medium $=101-400$ eggs per gram, high $>400$ eggs per gram. 
1265 persons, indicates a total number of 448 infected individuals. This figure compared to the 175 (13.8\%) individuals detected with a single KK smear, permits the conclusion that 274 (61.6\%) of the positives were not identified. These numbers improve with an increase of slides and samples-three smears of three different samples miss 153 (34\%) infected, but still remain far from an acceptable range, considering that our reference value is only an approximation to the real prevalence, which for sure is higher. Similar results were reported by Utzinger et al. (2002) and Booth et al. (2003) in a study carried out among schoolchildren from a rural area in Ivory coast.

As Fig. 1 reveals in a direct comparison of $1 \mathrm{KK}$ slide to the diagnostic 'gold' standard among positives of the experimental group, the distribution of prevalence, stratified by age groups, does not follow the well known pattern, which reaches a peak during childhood and for young adults, followed by a steady decrease with increasing age. Interestingly our data show high prevalence levels continuing during adult life. This finding is confirmed by other studies using statistic models, such as the Bayesian approach (Raso et al., 2007). The most probable explanation for this prolongation of high prevalence levels is the increase of sensitivity of the diagnostic method that allows identifying cases of schistosomiasis, which are not detected with a single smear. Given the fact that the absolute numbers of positives are much higher in the evaluation according to the diagnostic 'gold' standard (average of $44.3 \%$ from 10 to 50 years) compared to one slide (20.9\% from 10 to 30 years), the current strategy of the schistosomiasis control programme in Brazil, which is based on a single KK smear and the treatment of identified positives may be reconsidered. According to WHO (1993), mass treatment, without prior stool examination should be considered whenever prevalence reaches levels of $50 \%$ or higher. This approach may seem to be contradictive in this context, but our data show that even in areas of "so-called" low prevalence - that means overall prevalence is calculated based on one KK slide - specific age groups reach very high prevalence levels. Mass treatment of these specific age groups, which may be called targeted mass treatment, offers at least in theory a solution to the dilemma that many positives escape in the diagnostic process in these so-called low prevalence areas and consequently, by not receiving treatment, contribute significantly to the maintenance of the disease's transmission and are at risk to develop, although in milder form, chronic schistosomiasis caused pathologies. Putting this strategy into practice by applying it to our setting reveals that 301 positives of the age group from 10 to 50 would be treated directly, and together with 50 , which are detected through stool examination based on one KK slide of a single sample in the remaining age groups, sum a total number of 351 positive detected. Those are 176 positives more than by screening the whole population with a single KK smear, which identifies only 175 positive individuals in the total population for treatment. Nevertheless there remain, according to the prevalence figures of the diagnostic 'gold' standard, 107 positives, consisting of 30 in the age group under 10 and 77 in the age group over 50 , unidentified and untreated. One also may argue that a considerable number of uninfected individuals are submitted to unnecessary treatment. In our setting and according to the strategy 394 negative persons would receive chemotherapy. Although praziquantel is considered a safe drug, causing few and mainly mild side effects, a small risk remains to harm a healthy person. In order to assist policy makers in the field of public health in the decision-making process to use this drug under such circumstances, the responsibility lies not only with experts such as epidemiologists, parasitologists, pharmacists and medical doctors, but involves also the opinion of ethical boards and the proper community. Another issue in this context is the financial aspect. The direct costs in our setting consist in expenditure for the "traditional" approach of 1265 times the price of a KK smear and 175 times the price of treatment with praziquantel. The cost estimates for the suggested strategy include 570 times the price of a KK smear and 745 times the price of treatment with praziquantel resulting in a cost reduction by approximately $20 \%$ and an increase of positives treated by $100 \%$. Although these data show significant improvements in cost-effectiveness and in the efficacy of treating positives, they have to be seen with caution, because the estimates are made, based on the results of our setting. Therefore, more data of similar and different settings applying high standards of reference prevalence are necessary and welcomed, in order to validate our data before application on a larger scale. Nevertheless these new approach points into a possible direction how the efficacy of control programmes in low endemic areas can be improved by using tools of diagnostic and for treatment, which are cheap, easy and safe to use under field conditions. These considerations gain even more importance whenever the main objective of such programmes is switched from morbidity to transmission control.

The issue of infection intensity is more complex than the estimates of prevalence and more susceptible to bias. For the calculation of the infection intensity a simple qualitative analysis of faecal material is not enough. A quantitative evaluation is necessary for the calculation of epg. This requires a more sophisticated diagnostic approach. Fortunately, the KK technique meets these requirements, besides its well-known limitations and lack of reliability in areas of low transmission and consequently low infection intensity (Kongs et al., 2001; Rabelo and Enk, 2006). Different methodologies used for calculation of the mean infection intensity at individual and population level cause variations in results of the mean egg count, even within the same individual or population and makes comparisons between different populations very difficult (Fulford, 1994; Mutapi et al., 2003). In this study the main focus is on the impact of increasing number of slides and samples on infection intensity. According to our data a decrease of infection intensity goes clearly hand in hand with the increase of slides and samples. This decrease is based on the inclusion of individuals with low infection intensity and an indirect consequence of the increased sensibility of the diagnostic method. This dilution effect was found for the overall infection intensity on population level and on age group level.

The evaluation of the effect of increased number of slides and sample size on the infection categories low, medium and high, of the same 42 positive individuals reveals an increase of the number of positive individuals in the category of medium infections. In this case the daily variation of egg output, as extensively documented in the scientific literature (Barreto et al., 1990; Engels et al., 1997; Kongs et al., 2001) seems to be responsible. It causes randomly on the day when the stool sample is taken a variation of individual egg output and causes in this way the migration of positive individuals among infection intensity categories as described in Table 4.

The variation in the number of positives as shown in Table 3 is caused by a combination of the above-described effects. The increase of number in the category of light infections is mainly based on newly detected individuals and the increase of number in the category of moderate infections and the decrease of heavy infection intensity are result of migration.

Looking into the figures of age-dependent infection intensity, children show the highest mean egg count. This can be explained by the interaction of three factors. Firstly, the immune system of children in this age did not have enough time to develop a required immunity against schistosomiasis, which helps to control infection intensity as it is the case in adults. Secondly, children have a higher risk to become infected because of their physical activities, which increases the probability to get in contact with contaminated water. Thirdly, the simple fact that, given the same worm burden 
as an adult, the amount of eggs produced is distributed in a smaller volume of faecal material. Therefore, eggs are indirectly concentrated in the stool of children and a higher egg count is found if compared to the same amount of stool examined of an adult with the same worm burden. In the comparison of $1 \mathrm{KK}$ slide with 10 of three samples the infection intensity was reduced from 251 to 58 epg (the high egg count of 251 epg has to be seen with caution and is probably biased because of the small sample size in this age group. Nonetheless, and more importantly, all children with medium and high infection intensity were already detected with one single KK slide. As it is the case in this age group, all the others showed a decrease of infection intensity with an increase of slides and samples examined. This indicates that infection intensity is overestimated by $1 \mathrm{KK}$ slide in comparison to 10 slides of three samples. In general terms the impact of this decreasing effect in infection intensity on morbidity control programmes is not so easy to evaluate, because, as mentioned before, the infection intensity itself is complicated to calculate and depends on various factors, which influence it in accordance to the setting. Cautiously the assumption can be made that morbidity control programmes seem not to be as successful as initially thought, because they bring overestimated infection levels only to overestimated lower levels, when in reality considerable morbidity is already caused on low levels of infection intensity, which is confirmed by data from autopsies and by ultrasound (de Vlaas and Gryseels, 1992). A recent increase of reports about neuroschistosomiasis in Brazil confirms this assumption and leaves more doubts about the success of morbidity control programmes (Nobre et al., 2001; Ferrari et al., 2004; Nascimento-Carvalho and Moreno-Carvalho, 2005). All this together demonstrates that the relation between infection intensity and morbidity is not as straightforward as assumed, but is considered as such in many evaluations of control programmes, leading to an overestimation of the success of these programmes.

In conclusion our data confirm and reinforce that prevalence is underestimated and infection intensity is overestimated, if evaluated by a single KK smear. The findings provide evidence that indicate possible directions in which strategies for the control of schistosomiasis in low transmission areas could go. In the case of Brazil, decades after the introduction of a nationwide schistosomiasis control programme, which is mainly based on chemotherapy, major achievements are reported (Katz, 1998). Mortality rates and hospital admissions due to schistosomiasis were reduced by $50.0 \%$. Morbidity, measured indirectly by detection of the hepatosplenic form of the disease also decreased. These control efforts reduced prevalence and infection intensity, so that many of former high endemic areas were transformed into areas of low transmission. But as our study shows estimates for prevalence in this so-called low endemic areas may remain unexpectedly high, depending on the sensitivity of the diagnostic method, and real figures for prevalence are for sure even more elevated. This also demonstrates that the control programme currently in use has reached its limits of efficacy and efficiency. New strategies have to be elaborated which identify positive individuals undetected under the current diagnostic approach, in order to ensure the continuing success of the control programme. Under the current situation, considering especially the availability, or better non-availability of sensitive, sustainable, simple and field proven diagnostic tools, the choice for transmission control is targeted mass treatment, covering a wide range of the population with chemotherapy as described above.

\section{Acknowledgements}

We thank Aureo Almeida de Oliveira, senior technician of the Laboratory of Schistosomiasis, René Rachou Research Cen-
ter/Oswaldo Cruz Foundation, for his valuable contribution in realizing the fieldwork and examining the stool samples. We are grateful for the excellent cooperation of the technical team of the Secretary of Health in the State of Minas Gerais, Section Governador Valadares.

This work obtained financial support from the Fundação de Amparo a Pesquisa do Estado de Minas Gerais, Brazil and Conselho National de Pesquisa.

\section{References}

Barreto, M.L., Smith, D.H., Sleigh, A.C., 1990. Implications of faecal egg count variation when using the Kato-Katz method to assess Schistosoma mansoni infections. Trans. R. Soc. Trop. Med. Hyg. 84, 554-555.

Blagg, W., Schoegel, E.L., Mansour, N.S., Khalat, G.I., 1955. A new concentration technique for the demonstration of protozoa and helminth eggs in feces. Am. J. Trop. Med. Hyg. 4, 23-28.

Booth, M., Vounatsou, P., N'goran, E.K., Tanner, M., Utzinger, J., 2003. The influence of sampling effort and the performance of the Kato-Katz technique in diagnosing Schistosoma mansoni and hookworm co-infections in rural Côte d'Ivoire. Parasitology 127, 525-531.

Chitsulo, L., Engels, D., Montresor, A., Savioli, L., 2000. The global status of schistosomiasis and its control. Acta Trop. 77, 41-51.

de Vlaas, S.J., Gryseels, B., 1992. Underestimation of Schistosoma mansoni prevalences. Parasitol. Today 8, 274-277.

Engels, D., Sinzinkayo, E., de Vlas, S.J., Gryseels, B., 1996. Intraspecimen faecal egg count variation in Schistosoma mansoni infection. Am. J. Trop. Med. Hyg. 54, 319-324.

Engels, D., Sinzinkayo, E., de Vlas, S.J., Gryseels, B., 1997. Day-to-day egg count fluctuation in Schistosoma mansoni infection and its operational implications. Am. J. Trop. Med. Hyg. 57, 571-577.

Feldmeier, H., Poggensee, G., 1993. Diagnostic techniques in schistosomiasis control. A review. Acta Trop. 52, 205-220.

Ferrari, T.C., Moreira, P.R., Cunha, A.S., 2004. Spinal cord schistosomiasis: a prospective study of 63 cases emphasizing clinical and therapeutical aspects. J. Clin. Neurosci. 11, 246-253.

Fulford, A.J.C., 1994. Dispersion and bias: can we trust geometric means? Parasitol. Today $10,446-448$.

Kato, K., Miura, M., 1954. Comparative examinations Jap J Parasitol 3:35 (Japanese text). Apud Martin L.K., Beaver P.C., 1968. Evaluation of Kato thick-smear technique for quantitative diagnosis of helminth infections. Am. J. Trop. Med. Hyg. $17,382-391$.

Katz, N., 1998. Schistosomiasis control in Brazil. Mem. Inst. Oswaldo Cruz 93, 3335.

Katz, N., Chaves, A., Pellegrino, J., 1972. A simple device for quantitative stool thicksmear technique schistosomiasis mansoni. Rev. Inst. Med. Trop. São Paulo 14, 397-400.

Katz, N., Peixoto, S.V., 2000. Critical analysis of the estimated number of schistosomiasis mansoni carriers in Brazil. Rev. Soc. Bras. Med. Trop. 33, 303-308.

King, C.H., Dickman, K., Tisch, D.J., 2005. Reassessment of the coast of chronic helmintic infection: a meta-analysis of disability-related outcomes in endemic schistosomiasis. Lancet 365, 1561-1569.

Kongs, A., Marks, G., Verle, P., Van det Stuyft, P., 2001. The unreliability of the KatoKatz technique limits its usefulness for evaluation of $S$. mansoni infections. Trop. Med. Int. Health 6, 163-169.

Landis, J.R., Koch, G.G., 1977. The measurement of observer agreement for categorical data. Biometrics 33, 159-174.

Mutapi, F., Gryseels, B., Roddam, A., 2003. On the calculation of intestinal schistosome infection intensity. Acta Trop. 87, 225-233.

Nascimento-Carvalho, C.M., Moreno-Carvalho, O.A., 2005. Neuroschistosomiasis due to Schistosoma mansoni: a review of pathogenesis, clinical syndromes and diagnostic approaches. Rev. Inst. Med. Trop. Sao Paulo 47, 179-184.

Nobre, V., Silva, L.C., Ribas, J.G., Rayes, A., Serufo, J.C., Lana-Peixoto, M.A., Marinho, R.F., Lambetucci, J.R., 2001. Schistosomal myeloradiculopathy due to Schistosoma mansoni: report on 23 cases. Mem. Oswaldo Cruz 96, 137-141.

Passos, A.D.C., Amaral, R.S., 1998. Esquistossomose mansônica: aspectos epidemiológicos e de controle. Rev. Soc. Brasil Med. Trop. 31, 61-74.

Rabelo, A.L.T., Enk, M.J., 2006. Working Paper 8: progress towards the detection of schistosomiasis. Report of the Scientific Working Group on Schistosomiasis. WHO Publ., Geneva, pp. 67-72.

Rabelo, A.L.T., Rocha, R.S., Oliveira, J.P.M., Katz, N., Lambertucci, J.R., 1992. Stool examination and rectal biopsy in the diagnosis and therapeutical evaluation of schistosomiasis mansoni. Rev. Inst. Med. Trop. São Paulo 34, 601608

Raso, G., Vounatsou, P., McManus, D.P., N'Goran, E.K., Utzinger, J., 2007. A Bayesian approach to estimate the age-specific prevalence of Schistosoma mansoni and implications for schistosomiasis control. Int. J. Parasitol. 37, 1491-1500.

Steinmann, P., Keiser, J., Bos, R., Tanner, M., Utzinger, J., 2006. Schistosomiasis and water resources development: systematic review, meta-analysis and estimates of people at risk. Lancet Infect. Dis. 6, 411-425.

Utzinger, J., Booth, M., N'Goran, E.K., Muller, I., Tanner, M., Lengeler, C., 2001. Relative contribution of day-to-day and intra-specimen variation in faecal egg counts of 
Schistosoma mansoni before and after treatment with praziquantel. Parasitology $122,537-554$.

Utzinger, J., Vounatsou, P., N'Goran, E.K., Tanner, M., Booth, M., 2002. Reduction in the prevalence and intensity of hookworm infections after praziquantel treatment for schistosomiasis infection. Int. J. Parasitol. 32, 759-765.
WHO, 1993. The control of schistosomiasis. Technical Report Series, 830. World Health Organization, Geneva.

WHO, 1994. Bench Aids for the Diagnosis of Intestinal Parasites. World Health Organization, Geneva. 